\title{
CROSSED PRODUCT OF CYCLIC GROUPS
}

\author{
ANA-LOREDANA AGORE AND DRAGOŞ FRĂ ŢILA $\breve{~}$
}

\begin{abstract}
All crossed products of two cyclic groups are explicitly described using generators and relations. A necessary and sufficient condition for an extension of a group by a group to be a cyclic group is given.
\end{abstract}

\section{INTRODUCTION}

One of the most frequently used results in elementary number theory is the famous ancient Chinese Remainder Theorem. The Chinese Remainder theorem can be restated in an abstract and elegant language of group theory as follows: the direct product $H \times G$ of two groups is a cyclic group iff the groups are finite, cyclic of coprime orders. The direct product $H \times G$ is the trivial example of an extension of a group $H$ by a group $G$, that is there exists an exact sequence of groups:

$$
1 \longrightarrow H \stackrel{i_{H}}{\longrightarrow} H \times G \stackrel{\pi_{G}}{\longrightarrow} G \longrightarrow 1
$$

It is therefore natural and tempting to consider the most general problem:

Problem 1: Let $(E, i, \pi)$ be an extension of $H$ by $G$ : i.e. $E$ is a group, $i: H \rightarrow E$ and $\pi: E \rightarrow G$ are morphisms of groups such that the sequence

$$
1 \longrightarrow H \stackrel{i}{\longrightarrow} E \stackrel{\pi}{\longrightarrow} G \longrightarrow 1
$$

is exact. Give a necessary and sufficient condition for the group $E$ to be cyclic.

The main theorem of the paper (Theorem 3.8) gives a complete answer to the above question. From this point of view Theorem 3.8 can be considered as an interesting and non-trivial generalization of the Chinese Remainder Theorem.

To obtain this result we will go through the following steps: we will do a survey of the famous "extension problem" of Hölder [6], then we will work in an equivalent way with the crossed systems instead of exact sequences and in the end we will explicitly compute all the symmetric, normalized 2-cocycles for two fixed cyclic groups and the (crossed)twisted products associated.

The extension problem was first stated by Hölder [6]. A recent survey and new results related to the extension problem are obtained in 3. In particular, crossed products arise naturally when dealing with group extensions. [3, Corollary 1.8] is another formulation

2000 Mathematics Subject Classification. 20B05, 20B35, 20D06, 20 D40.

The authors where supported by CNCSIS grant 24/28.09.07 of PN II "Groups, quantum groups, corings and representation theory". 
of Schreier theorem and shows that the existence of an extension of $H$ by $G$ is equivalent to the existence of a normalized crossed system $(H, G, \alpha, f)$, where $\alpha: G \rightarrow \operatorname{Aut}(H)$ is a weak action and $f: G \times G \rightarrow H$ is an $\alpha$-cocycle. The classical extension problem of Hölder was restated in [3, Problem 1] in a computational manner as follows:

Problem 2: Let $H$ and $G$ be two fixed groups. Describe all normalized crossed systems $(H, G, \alpha, f)$ and classify up to isomorphism all crossed products $H \#_{\alpha}^{f} G$.

The first notable result regarding the extension problem was given by O. L. Hölder (Theorem 2.1), who uses generators and relations to describe all crossed products of two finite cyclic groups. In the section 2 of the paper we complete the structure and we shall describe all crossed products of two cyclic groups (not necesary finite) using generators and relations: see Theorem 2.2. Theorem 2.3 and Theorem 2.4. Related to Problem 2 another question arise:

Problem 3: Let $\Lambda$ be a class of groups. Find necessary and sufficient conditions for $(H, G, \alpha, f)$ such that the crossed product $H \#_{\alpha}^{f} G$ belongs to $\Lambda$.

In [3, Corollary 1.15] a complete answer is given for the above problem in the case of

abelian groups: the crossed product $H \#_{\alpha}^{f} G$ is an abelian group if and only if $H$ and $G$ are abelian groups, $\alpha$ is the trivial action and $f$ is a symmetric 2 -cocycle.

The present paper deals with this problem in the case of cyclic groups. In the first section we recall the construction and fundamental properties of crossed product of groups. In section 2 we describe crossed products between all types of cyclic groups. Using the aforementioned results, in Section 3 we find necessary and sufficient conditions for a crossed product to be a cyclic group (Theorem [3.8) which is the main result of this paper.

\section{Preliminaries}

Let us fix the notations that will be used throughout the paper. $C_{n}$ will be a cyclic group of order $n$ generated by $a: C_{n}=\left\{1, a, a^{2}, \cdots, a^{n-1}\right\}$ and $C_{g}=\left\{g^{k} \mid k \in \mathbb{Z}\right\}$ will denote a cyclic infinite group. Let $H$ and $G$ be two groups. Aut $(H)$ denotes the group of automorphisms of a group $H$ and $Z(H)$ the center of $H$. A map $f: G \times G \rightarrow H$ is called symmetric if $f\left(g_{1}, g_{2}\right)=f\left(g_{2}, g_{1}\right)$ for any $g_{1}, g_{2} \in G$. For a map $\alpha: G \rightarrow \operatorname{Aut}(H)$ we shall use the notation

$$
\alpha(g)(h)=g \triangleright h
$$

for all $g \in G$ and $h \in H$.

The maps $\alpha$ and $f$ are called trivial if $g \triangleright h=h$ for all $g \in G$ and $h \in H$, respectively $f\left(g_{1}, g_{2}\right)=1$ for all $g_{1}, g_{2} \in G$.

Definition 1.1. A crossed system of groups is a quadruple $(H, G, \alpha, f)$, where $H$ and $G$ are two groups, $\alpha: G \rightarrow \operatorname{Aut}(H)$ and $f: G \times G \rightarrow H$ are two maps such that the following compatibility conditions hold:

$$
\begin{aligned}
g_{1} \triangleright\left(g_{2} \triangleright h\right) & =f\left(g_{1}, g_{2}\right)\left(\left(g_{1} g_{2}\right) \triangleright h\right) f\left(g_{1}, g_{2}\right)^{-1} \\
f\left(g_{1}, g_{2}\right) f\left(g_{1} g_{2}, g_{3}\right) & =\left(g_{1} \triangleright f\left(g_{2}, g_{3}\right)\right) f\left(g_{1}, g_{2} g_{3}\right)
\end{aligned}
$$


for all $g_{1}, g_{2}, g_{3} \in G$. The crossed system $\Gamma=(H, G, \alpha, f)$ is called normalized if $f(1,1)=1$. The map $\alpha: G \rightarrow \operatorname{Aut}(H)$ is called a weak action and $f: G \times G \rightarrow H$ is called an $\alpha$-cocycle.

If $(H, G, \alpha, f)$ is a normalized crossed system then [3, Lemma 1.2]

$$
f(1, g)=f(g, 1)=1 \quad \text { and } \quad 1 \triangleright h=h
$$

for any $g \in G$ and $h \in H$.

Let $H$ and $G$ be groups, $\alpha: G \rightarrow \operatorname{Aut}(H)$ and $f: G \times G \rightarrow H$ two maps. Let $H \#_{\alpha}^{f} G:=H \times G$ as a set with a binary operation defined by the formula:

$$
\left(h_{1}, g_{1}\right) \cdot\left(h_{2}, g_{2}\right):=\left(h_{1}\left(g_{1} \triangleright h_{2}\right) f\left(g_{1}, g_{2}\right), g_{1} g_{2}\right)
$$

for all $h_{1}, h_{2} \in H, g_{1}, g_{2} \in G$. Then [3, Theorem 1.3] $\left(H \#_{\alpha}^{f} G, \cdot\right)$ is a group with the unit $1_{H \#_{\alpha}^{f} G}=(1,1)$ if and only if $(H, G, \alpha, f)$ is a normalized crossed system. In this case the group $H \#_{\alpha}^{f} G$ is called the crossed product of $H$ and $G$ associated to the crossed system $(H, G, \alpha, f)$.

The following [3, Examples 1.5] are basic examples of special cases of a crossed product of two groups.

Examples 1.2. 1. Let $H$ and $G$ be two groups and $\alpha, f$ be the trivial maps. Then $\Gamma=(H, G, \alpha, f)$ is a crossed system called the trivial crossed system. The crossed product $H \#_{\alpha}^{f} G=H \times G$ is the direct product of $H$ and $G$.

2. Let $H$ and $G$ be two groups and $f: G \times G \rightarrow H$ the trivial map. Then $(H, G, \alpha, f)$ is a crossed system if and only if $\alpha: G \rightarrow \operatorname{Aut}(H)$ is a morphism of groups. In this case the crossed product $H \#_{\alpha}^{f} G=H \ltimes_{\alpha} G$, the semidirect product of $H$ and $G$.

3. Let $H$ and $G$ be two groups and $\alpha: G \rightarrow \operatorname{Aut}(H)$ the trivial action. Then $(H, G, \alpha, f)$ is a crossed system if and only if $\operatorname{Im}(f) \subseteq Z(H)$ and

$$
f\left(g_{1}, g_{2}\right) f\left(g_{1} g_{2}, g_{3}\right)=f\left(g_{2}, g_{3}\right) f\left(g_{1}, g_{2} g_{3}\right)
$$

for all $g_{1}, g_{2}, g_{3} \in G$, that is $f: G \times G \rightarrow Z(H)$ is a 2-cocycle. The crossed product $H \#_{\alpha}^{f} G$ associated to this crossed system will be denoted by $H \times{ }^{f} G$ and was called in 3 the twisted product of $H$ and $G$ associated to the 2-cocycle $f: G \times G \rightarrow Z(H)$. Explicitly, the multiplication of a twisted product of groups $H \times{ }^{f} G$ is given by the formula:

$$
\left(h_{1}, g_{1}\right) \cdot\left(h_{2}, g_{2}\right):=\left(h_{1} h_{2} f\left(g_{1}, g_{2}\right), g_{1} g_{2}\right)
$$

for all $h_{1}, h_{2} \in H, g_{1}, g_{2} \in G$.

The next well known theorem is the main application of the crossed product construction: it is a reconstruction theorem of a group from a normal subgroup and the quotient.

Theorem 1.3. Let $E$ be a group $H \unlhd E$ be a normal subgroup of $E$ and $G:=E / H$ be the quotient of $E$ by $H$. Then there exists two maps $\alpha: G \rightarrow \operatorname{Aut}(H)$ and $f: G \times G \rightarrow H$ such that $(H, G, \alpha, f)$ is a normalized crossed system and $E \cong H \#_{\alpha}^{f} G$ (isomorphism of groups). 
For complete proofs and further details we refer to [2], [3, Theorem 1.6] or [8].

\section{Crossed product of cyclic groups}

Our purpose in this section is to describe using generators and relations all crossed products between cyclic groups. As mentioned in the introduction, the first important result in literature for the first part of the extension problem was proved by Hölder himself [5, Theorem 12.9]. It describes the crossed product of two finite cyclic groups: for the sake of completeness we present bellow a short proof of this theorem.

Theorem 2.1. (Holder) A finite group $E$ is isomorphic to a crossed product $C_{n} \#_{\alpha}^{f} C_{m}$ if and only if $E$ is the group generated by two generators $a$ and $b$ subject to the relations

$$
a^{n}=1, \quad b^{m}=a^{i}, \quad b^{-1} a b=a^{j}
$$

where $i, j \in\{0,1, \cdots, n-1\}$ such that

$$
i(j-1) \equiv 0(\bmod n), \quad j^{m} \equiv 1(\bmod n)
$$

We denote this group by $C_{n} \#_{i}^{j} C_{m}$.

Proof. Assume first that $\mathrm{E}$ is isomorphic to a crossed product $C_{n} \#_{\alpha}^{f} C_{m}$. Hence $C_{n} \unlhd E$ and $E / C_{n} \simeq C_{m}$. It follows that $C_{n}=<a \mid a^{n}=1>\unlhd E$ and there exists $b \in E$ such that $E / C_{n}=\left\{C_{n}, b C_{n}, \ldots, b^{m-1} C_{n}\right\}$ and $b^{m} \in C_{n}$. That is, there exists $i \in\{0,1, \ldots, n-1\}$ such that:

$$
b^{m}=a^{i}
$$

Since $C_{n} \unlhd E$ we obtain that $b^{-1} a b \in C_{n}$ and so there exists $j \in\{0,1 \ldots, n-1\}$ such that:

$$
b^{-1} a b=a^{j}
$$

A direct computation shows that:

$$
b^{-1} a^{i} b \stackrel{\text { 99 }}{=} b^{-1} b^{m} b=b^{m} \stackrel{\text { 99 }}{=} a^{i} \quad \text { and } \quad b^{-1} a^{i} b \stackrel{100}{=} a^{i j}
$$

It follows from here that $a^{i(j-1)}=1$ and so $i(j-1) \equiv 0(\bmod n)$. In a similar way we obtain:

$$
b^{-m} a b^{m} \stackrel{\text { 9) }}{=} a^{-i} a a^{i}=a \text { and } a^{j^{2}} \stackrel{\text { 10 }}{=}\left(b^{-1} a b\right)^{j}=b^{-1} a^{j} b \stackrel{\text { 10 }}{=} b^{2} a b^{2}
$$

and by induction : $b^{-m} a b^{m}=a^{j^{m}}$. Hence $a=a^{j^{m}}$, that is $j^{m} \equiv 1(\bmod n)$.

Conversely, assume that relations (7) and (8) hold. We need to show that $C_{n} \unlhd E$, that is $x a^{t} x^{-1} \in C_{n}$ for every $x \in E$ and $t \in\{0,1, \ldots, n-1\}$. Since $x \in E$ we have $x=x_{1} x_{2} \ldots x_{k}$ where $k \in \mathbb{N}, x_{s} \in\left\{a, b, a^{-1}, b^{-1}\right\}$ and $s \in\{0,1, \ldots, k\}$. We obtain that $g a^{t} g^{-1}=x_{1} x_{2} \ldots x_{k} a^{t}\left(x_{k}\right)^{-1} \ldots\left(x_{1}\right)^{-1}$. It is easy to see by a direct computation that $x_{k} a^{t}\left(x_{k}\right)^{-1} \in C_{n}$ for every $x_{k} \in\left\{a, b, a^{-1}, b^{-1}\right\}$ and so, by induction it follows that $g a^{t} g^{-1} \in C_{n}$. Hence $C_{n} \unlhd E$. In a similar way, it can be showed that every element of the group E can be written as $a^{p} b^{q}$ for $p, q \in \mathbb{Z}$. Hence $|E|=m n$ and so $\left|E / C_{n}\right|=m$, 
$E / C_{n}=\left\{C_{n}, C_{n} b, \ldots, C_{n} b^{m-1}\right\}$ that is, the group $E$ has a normal subgroup $C_{n}$ and $E / C_{n} \simeq C_{m}$. By Theorem 1.3, there exists $\left(C_{n}, C_{m}, \alpha, f\right)$ a crossed system such that $E \simeq C_{n} \#_{\alpha}^{f} C_{m}$.

Theorem 2.2. A group $E$ is isomorphic to a crossed product $C_{n} \#_{\alpha}^{f} C_{g}$ if and only if there exists $t \in \mathbb{Z},(t, n)=1$ such that $E \simeq<a, g \mid a^{n}=1, g^{-1} a g=a^{t}>$.

Proof. Suppose first that $E \simeq C_{n} \#_{\alpha}^{f} C_{g}$. Hence $C_{n} \unlhd E$ and $E / C_{n} \simeq C_{g}$. That is $E / C_{n}=\left\{g^{k} C_{n} \mid k \in \mathbb{Z}\right\}$. Since $C_{n} \unlhd E$ we obtain that $C_{n}=<a \mid a^{n}=1>\subseteq E$ and $g^{-1} a g \in C_{n}$. That is, there exists $t \in\{0,1, \ldots, n-1\}$ such that

$$
g^{-1} a g=a^{t}
$$

Suppose now that $(t, n)=d>1$. It follows from here that there exist $t_{1}, n_{1} \in N$ such that $t=d t_{1}, n=d n_{1}$ and $\left(t_{1}, n_{1}\right)=1$. From (11) we obtain $g^{-1} a^{n_{1}} g=a^{n t_{1}}=1$, that is $a^{n_{1}}=1$, which is a contradiction with $a$ having order $n$ and $n_{1}<n$. Hence $(t, n)=1$ and $E \simeq<a, g \mid a^{n}=1, g^{-1} a g=a^{t}>$.

Now let $E \simeq<a, g \mid a^{n}=1, g^{-1} a g=a^{t}>$ for some $t \in \mathbb{Z},(t, n)=1$. By Theorem 1.3 we only need to prove that $C_{n} \unlhd E$ and $E / C_{n} \simeq C_{g}$. For any $g^{\prime} \in E$ we have $g^{\prime}=x_{1} x_{2} \ldots x_{k}$, for some $k \in \mathbb{N}, x_{i} \in\left\{a, g, a^{-1}, g^{-1}\right\}, i \in\{1,2, \ldots, k\}$. That is, to prove that $C_{n} \unlhd E$ we only need to show that $g^{-1} a^{l} g \in C_{n}$ and $g a^{l} g^{-1} \in C_{n}$ for any $l \in \mathbb{Z}$. From (11) we obtain, by induction, that $g^{-1} a^{l} g=a^{t l} \in C_{n}$. Since $(t, n)=1$ there exist $\alpha, \beta \in \mathbb{Z}$ such that $\alpha t+\beta n=1$. We obtain from (111) that $a=g a^{t} g^{-1}$ and it follows from here that $a^{\alpha}=g a^{\alpha t} g^{-1}$. Since $g a^{\beta n} g^{-1}=1$ we obtain that $g a^{\alpha t+\beta n} g^{-1}=a^{\alpha}$, that is $g a g^{-1}=a^{\alpha}$. It follows from here that $g a^{l} g^{-1}=a^{\alpha l}$ for any $l \in \mathbb{Z}$. Hence $C_{n} \unlhd E$. It follows by a simple calculation that every element $g^{\prime} \in E$ can be written as $g^{p} a^{q}$ for some $p, q \in \mathbb{Z}$. That is $g C_{n}=g^{p} a^{q} C_{n}={ }^{p} C_{n}$. Hence $E / C_{n} \subseteq C_{g}$. Now suppose that there exist $\alpha, \beta \in \mathbb{Z}, \alpha \neq \beta$ such that $g^{\alpha} C_{n}=g^{\beta} C_{n}$, that is $g^{\alpha-\beta}=a^{\gamma}$ for some $\gamma \in\{0,1, \ldots, n-1\}$. It follows from here that $g^{(\alpha-\beta) n}=a^{\gamma n}=1$ which is a contradiction with $C_{g}$ being an infinite cyclic group. Hence $E / C_{n} \simeq C_{g}$.

Theorem 2.3. A group $E$ is isomorphic to a crossed product $C_{g} \#_{\alpha}^{f} C_{n}$ if and only if:

(i) $E \simeq<g, h \mid g h=h g, h^{n}=g^{t}, t \in \mathbb{Z}>$ for $n$ odd;

(ii) $E \simeq<g, h \mid g h=h g, h^{n}=g^{t}, t \in \mathbb{Z}>$ or $E \simeq<g, h \mid h^{n}=1, g h g=h>$ for $n$ even.

Proof. Suppose first that $E \simeq C_{g} \#_{\alpha}^{f} C_{n}$. Hence $C_{g} \unlhd E$ and $E / C_{g} \simeq C_{n}$. That is, there exists $h \in E$ such that $E / C_{g}=\left\{C_{g}, h C_{g}, \ldots, h^{n-1} C_{g}\right\}$ and $h^{n} \in C_{g}$. Hence there exists $t \in \mathbb{Z}$ such that $h^{n}=g^{t}$. Since $C_{g} \unlhd E$ we obtain $h^{-1} g h \in C_{g}$, that is $h^{-1} g h=g^{s}$ for some $s \in \mathbb{Z}$. It follows that $h^{-1} g^{t} h=g^{s t}$ and using $h^{n}=g^{t}$ we obtain $g^{t s}=g^{t}$ that is $g^{t(s-1)}=1$. Since $C_{g}$ is a infinite cyclic group we must have $t(s-1)=0$. Using again $h^{-1} g h=g^{s}$ we obtain $h^{-1} g^{s} h=g^{s^{2}}$, that is $h^{-2} g h^{2}=g^{s^{2}}$ and by induction $h^{-n} g h^{n}=g^{s^{n}}$. Thus, from $h^{n}=g^{t}$ we obtain $g^{s^{n}-1}=1$ and since $C_{g}$ is an infinite cyclic group we must have $s^{n}=1$. Therefore if $n$ is odd $E \simeq<g, h \mid g h=h g, h^{n}=g^{t}>$ for some $t \in \mathbb{Z}$ and if $n$ is even $E \simeq<g, h \mid g h=h g, h^{n}=g^{t}>$ for some $t \in \mathbb{Z}$ or $E \simeq<g, h \mid h^{n}=1, g h g=h>$. 
We assume now that $E \simeq<g, h \mid g h=h g, h^{n}=g^{t}, t \in \mathbb{Z}>$. Since $E$ is abelian $C_{g} \unlhd E$. $E / C_{g}=\left\{g^{\prime} C_{g} \mid g^{\prime} \in E\right\}$ and since every element $g^{\prime} \in E$ can be written as $g^{\prime}=h^{p} g^{q}$ we obtain that $g^{\prime} C_{g}=h^{p} g^{q} C_{g}=h^{p} C_{g}$ that is $E / C_{g} \subseteq\left\{C_{g}, h C_{g}, \ldots, h^{n-1} C_{g}\right\} \simeq C_{n}$. Now suppose that there exists $\alpha, \beta \in\{0,1, \ldots, n-1\}, \alpha>\beta$, such that $h^{\alpha} C_{g}=h^{\beta} C_{g}$ that is $h^{\alpha-\beta}=g^{\gamma}$ for some $\gamma \in \mathbb{Z}$. Since $\alpha-\beta<n$ we obtain a contradiction with $h^{n}=g^{t}$. Hence $E / C_{g} \simeq C_{n}$ and by Theorem 1.3 there exists $\left(C_{g}, C_{n}, \alpha, f\right)$ a crossed system such that $E \simeq C_{g} \#_{\alpha}^{f} C_{n}$.

Suppose now that $n$ is even and $E \simeq<g, h \mid h^{n}=1, g h g=h>$. By Theorem 1.3 we only need to prove that $C_{g} \unlhd E$ and $E / C_{g} \simeq C_{n}$. For any $g^{\prime} \in E$ we have $g^{\prime}=x_{1} x_{2} \ldots x_{k}$ for some $k \in \mathbb{N}, x_{i} \in\left\{g, h, g^{-1}, h^{-1}\right\}$ and $i \in\{1,2, \ldots, k\}$. That is, to prove that $C_{g} \unlhd E$ we only need to show that $h g^{l} h^{-1} \in C_{g}$ and $h^{-1} g^{l} h \in C_{g}$ for any $l \in \mathbb{Z}$. Since $h^{-1} g h=g^{-1}$ we obtain, by induction, that $h^{-1} g^{l} h=g^{-l} \in C_{g}$. Also from $h g^{l} h^{-1}=h^{-n+1} g^{l} h^{n-1}=\left(h^{-1}\right)^{n-1} g^{l} h^{n-1}=\left(h^{-1}\right)^{n-2} g^{-l} h^{n-2}$ we obtain by induction $h g^{l} h^{-1}=g^{-l} \in C_{g}$. Hence $C_{g} \unlhd E . E / C_{g}=\left\{g^{\prime} C_{g} \mid g^{\prime} \in E\right\}$ and since any element $g^{\prime} \in E$ can be written as $h^{p} g^{q}$ for some $p, q \in \mathbb{Z}$ it follows from here that $g^{\prime} C_{g}=h^{p} g^{q} C_{g}=h^{p} C_{g}$. Hence $E / C_{g} \subseteq C_{n}$. Now suppose that there exist $\alpha, \beta \in\{0,1, \ldots, n-1\}, \alpha>\beta$ such that $h^{\alpha} C_{g}=h^{\beta} C_{g}$, that is $h^{\alpha-\beta}=g^{\gamma}$ for some $\gamma \in \mathbb{Z}$. It follows from here that $g^{n \gamma}=\left(h^{n}\right)^{\alpha-\beta}=1$ and since $C_{g}$ is an infinite cyclic group we must have $n \gamma=0$, that is $\gamma=0$. Hence $h^{\alpha-\beta}=1$ which is a contradiction since the order of $h$ is $n$ and $0<\alpha-\beta<n$. Therefore $E / C_{g}=C_{n}$.

Theorem 2.4. A group $E$ is isomorphic to a crossed product $C_{g_{1}} \#_{\alpha}^{f} C_{g_{2}}$ if and only if $E \simeq<g_{1}, g_{2} \mid g_{1} g_{2}=g_{2} g_{1}>$ or $E \simeq<g_{1}, g_{2} \mid g_{1} g_{2} g_{1}=g_{2}>$.

Proof. Suppose first that $E \simeq C_{g_{1}} \#_{\alpha}^{f} C_{g_{2}}$. Hence $C_{g_{1}} \unlhd E$ and $E / C_{g_{1}} \simeq C_{g_{2}}$. That is $E / C_{g_{1}}=\left\{g_{2}^{k} C_{g_{1}} \mid k \in \mathbb{Z}\right\}$ Since $C_{g_{1}} \unlhd E$ we obtain that $g_{2}^{-1} g_{1} g_{2} \in C_{g_{1}}$ and $g_{2} g_{1} g_{2}^{-1} \in C_{g_{1}}$. That is, there exist $s, t \in \mathbb{Z}$ such that

$$
g_{2}^{-1} g_{1} g_{2}=g_{1}^{t}
$$

and

$$
g_{2} g_{1} g_{2}^{-1}=g_{1}^{s}
$$

From (12) we obtain, that $g_{2}^{-1} g_{1}^{s} g_{2}=g_{1}^{s t}$. It follows from here, using (13), that $g_{1}^{s t}=$ $g_{1}$. Since $C_{g_{1}}$ is an infinite cyclic group, we obtain that $s t=1$, that is $(s, t) \in$ $\{(1,1),(-1,-1)\}$. Hence $E \simeq<g_{1}, g_{2} \mid g_{1} g_{2}=g_{2} g_{1}>$ or $E \simeq<g_{1}, g_{2} \mid g_{1} g_{2} g_{1}=g_{2}>$.

Conversely, if $E \simeq<g_{1}, g_{2} \mid g_{1} g_{2}=g_{2} g_{1}>$ then it is obvious that $E \simeq \mathbb{Z} \times \mathbb{Z} \simeq C_{g_{1}} \# C_{g_{2}}$ the crossed system being the trivial one.

Now let $E \simeq<g_{1}, g_{2} \mid g_{1} g_{2} g_{1}=g_{2}>$. Also by Theorem 1.3 we only need to prove that $C_{g_{1}} \unlhd E$ and $E / C_{g_{1}} \simeq C_{g_{2}}$. For any $g \in E$ we have $g=x_{1} x_{2} \ldots x_{k}$ for $k \in N$, $x_{i} \in\left\{g_{1}, g_{2}, g_{1}^{-1}, g_{2}^{-1}\right\}$ and $i \in\{1,2, \ldots, k\}$. Therefore, to prove $C_{g_{1}} \unlhd E$, we only need to show that $g_{2} g_{1}^{l} g_{2}^{-1} \in C_{g_{1}}$ and $g_{2}^{-1} g_{1}^{l} g_{2} \in C_{g_{1}}$ for any $l \in \mathbb{Z}$. From $g_{1} g_{2} g_{1}=g_{2}$ we obtain $g_{2} g_{1} g_{2}^{-1}=g_{1}^{-1}=g_{2}^{-1} g_{1} g_{2}$ and $g_{2} g_{1}^{l} g_{2}^{-1}=g_{1}^{-l}=g_{2}^{-1} g_{1}^{l} g_{2}$ for any $l \in \mathbb{Z}$. Hence $C_{g_{1}} \unlhd E$. In a similar way it can be shown that every element g of the group $E$ can be written as $g_{2}^{p} g_{1}^{q}$ for some $p, q \in \mathbb{Z}$. It follows from here that $g C_{g_{1}}=g_{2}^{p} g_{1}^{q} C_{g_{1}}=g_{2}^{p} C_{g_{1}}$. 
Hence $E / C_{g_{1}} \leq C_{g_{2}}$. Since any non trivial subgroup of an infinite cyclic group is infinite cyclic we obtain that $E / C_{g_{1}} \simeq C_{g_{2}}$ which finishes the proof.

\section{When is a crossed product a cyclic group?}

Our aim in the present section is to give a necessary and sufficient condition for a crossed product to be a cyclic group. For this it is necessary that both groups should be cyclic since any subgroup and any quotient of a cyclic group are cyclic groups. Hence the problem is reduced to decide which of the crossed products between two cyclic groups described in the previous section are cyclic groups and under what conditions.

It is obvious that the crossed product between a finite cyclic group $C_{n}$ and an infinite cyclic group $C_{g}$ described in Theorem 2.2 can not be a cyclic group since an infinite cyclic group does not have torsion elements. By the same argument we can conclude that the crossed product $<g, h \mid h^{n}=1, g h g=h>$ obtained in Theorem 2.3 can not be a cyclic group. Also, the crossed product between the two infinite cyclic groups described in Theorem 2.4 can not be a cyclic group because a nontrivial quotient of an infinite cyclic group must be finite.

Therefore, the only crossed products left to deal with are : $C_{n} \#_{i}^{j} C_{m}$ described in Theorem 2.1 and $<g, h \mid g h=h g, h^{n}=g^{t}, t \in \mathbb{Z}>$ from Theorem 2.3.

In what follows we investigate under which conditions these two crossed products are cyclic groups.

In order to prove our next result we need the following technical lemma:

Lemma 3.1. Let $m, n, i$ be rational integers so that $(m, n, i)=1$. Then there exists some $u, v, w \in \mathbb{Z}$ such that $u m+v i+w n=1$ and $(m, v)=1$, where $(r, s)$ denotes the greatest common divisor of the integers $r$ and $s$.

Proof. Let $d=(m, n)$. Then $(d, i)=1$. Let $m^{\prime} \mid m$ be so that $\left(m^{\prime}, d\right)=1$ and $m^{\prime} d$ contains all the prime factors of $m$. Using the Chinese Reminder theorem we can find $v \in \mathbb{Z}$ such that $d \mid v i-1$ and $m^{\prime} \mid v-1$ (if $m^{\prime}=1$ the last condition is trivially fulfilled). We observe that $(m, v)=1$ because all the prime divisors of $m$ are in $m^{\prime} d$ and $\left(m^{\prime} d, v\right)=1$.

Since $d=(m, n)$ there exist $u^{\prime}, w^{\prime} \in \mathbb{Z}$ such that $u^{\prime} m+w^{\prime} n=d$. From the way we chose $v$ it follows that there exists $r \in \mathbb{Z}$ such that $v i+r d=1$. Now put $u=r u^{\prime}, w=r w^{\prime}$. From the above we have $v i+r\left(u^{\prime} m+w^{\prime} n\right)=1$, thus $v i+u m+w n=1$.

Proposition 3.2. A crossed product $E=C_{n} \#_{i}^{j} C_{m}$ is a cyclic group if and only if $j=1$ and $(m, n, i)=1$.

Proof. We know from Theorem 2.1 that $E$ has a presentation of the form

$$
E=<a, b \mid a^{n}=1, b^{m}=a^{i}, b^{-1} a b=a^{j}>.
$$

Suppose first that $E$ is a cyclic group. It follows from here that $j=1$ since every cyclic group is abelian. If $E$ is cyclic then there exist some $u, v \in \mathbb{Z}$ such that $E=\left\langle a^{u} b^{v}\right\rangle$. $a^{u} b^{v}$ has order $m n$, hence $\left(a^{u} b^{v}\right)^{m}$ has order $n$. It is well known that in a cyclic group for any divisor of the order of the group there exists a unique subgroup of that order, thus 
$\left.<\left(a^{u} b^{v}\right)^{m}\right\rangle=\langle a\rangle$ and therefore there exists some $k \in \mathbb{Z}$ such that $\left(a^{u k} b^{v k}\right)^{m}=a$. Using the relation $b^{m}=a^{i}$ and the fact that $a$ has order $n$ we obtain that $u k m+v k i-1$ is divisible by $n$, that is $(m, n, i)=1$.

For the converse we will use the previous lemma and we obtain that there exist $u, v, w \in \mathbb{Z}$ such that $u m+i v+w n=1$ and $(m, v)=1$. We will prove that $a^{u} b^{v}$ has order $m n$ in $E$ and that finishes the proof. For this it is enough to prove that $a, b \in\left\langle a^{u} b^{v}\right\rangle$. By a simple calculation we get: $\left(a^{u} b^{v}\right)^{m}=a^{u m} a^{v i}=a^{u m+v i}=a^{1-w n}=a$, that is $\left.a \in<a^{u} b^{v}\right\rangle$. Since $(m, v)=1$, there exists $l \in \mathbb{Z}$ such that $m \mid v l-w$. Now let $k=i+\ln$. Finally $\left(a^{u} b^{v}\right)^{k}=\left(a^{u} b^{v}\right)^{i}\left(a^{u} b^{v}\right)^{l n}=b^{u m} b^{v i} b^{v l n}=b^{u m+v i+v l n}=b^{1-w n+v l n}=b b^{n(v l-w)}=$ $b$ because $n(v l-w)$ is divisible by $m n$ and $|E|=m n$. Hence $b \in<a^{u} b^{v}>$.

Proposition 3.3. The group $E=<g, h \mid h^{n}=g^{t}, h g=g h>$ where $n \geq 2, t \in \mathbb{Z}$ is cyclic if and only if $(n, t)=1$.

Proof. Denote by $d=(n, t)$ and by $Z_{n, t}=\mathbb{Z}+\frac{t}{n} \mathbb{Z}$ which is an abelian group and is isomorphic to $(n, t) \mathbb{Z} \simeq \mathbb{Z}$ by the morphism $u \mapsto n u$.

Define $\theta: E \rightarrow Z_{n, t}$ by $h \mapsto \frac{t}{n}$ and $g \mapsto 1$. It is easy to see that this is a morphism of groups and moreover it is surjective. In order to have $E$ isomorphic to $\mathbb{Z}$ (i.e. $E$ cyclic infinite), $\theta$ must be an isomorphism, otherwise we get a surjective endomorphism of $\mathbb{Z}$ which is not injective and this is impossible.

So $E \simeq \mathbb{Z}$ iff $\theta$ is injective and this happens iff $(n, t)=1$. Indeed $h^{r} g^{-s} \mapsto \frac{r t}{n}-s=0 \Leftrightarrow$ $r t=n s \Leftrightarrow r=\frac{k n}{d}, s=\frac{k t}{d}, k \in \mathbb{Z}$ so $\operatorname{ker}(\theta)=\left\{\left(h^{\frac{n}{d}} g^{-\frac{t}{d}}\right)^{k}: k \in \mathbb{Z}\right\}$ and then $\operatorname{ker}(\theta)=0$ iff $h^{\frac{n}{d}}=g^{\frac{t}{d}}$, i.e. $d=1$.

Our next goal is to describe, in the language of crossed systems, all cyclic crossed products. That is, to identify the properties that $(H, G, \alpha, f)$ has to verify in order to obtain a cyclic crossed product $H \#_{\alpha}^{f} G$. As we already noticed, both $H$ and $G$ must be cyclic groups. Since $H \#_{\alpha}^{f} G$ is, in particular, an abelian group it follows from [3, Corrolary $1.15]$ that $\alpha$ must be trivial and $f$ a symmetric 2-cocycle. In order to find necessary and sufficient conditions on $f$ such that $H^{f} G$ is cyclic we describe bellow all the possible symmetric 2-cocycles.

For $m \geq 2$ and $n \geq 2$ or $n=\infty$ define $\Sigma_{m, n}=\left\{\phi: \mathbb{Z} \rightarrow \mathbb{Z}_{n}: \phi(0)=0, \phi(t+m)=\right.$ $\phi(t), \forall t \in \mathbb{Z}\}$ with the convention that $\mathbb{Z}_{\infty}=\mathbb{Z}$.

Proposition 3.4. The symmetric normalized 2-cocycles $f: C_{m} \times C_{m} \rightarrow C_{n}$ are in bijection with the set $\Sigma_{m, n}$.

Proof. Let $\phi \in \Sigma_{m, n}$ and consider $x$ a generator of $C_{m}$ and $a$ a generator of $C_{n}$.

Denote, $S_{k}^{\phi}=S_{k}=\phi(0)+\ldots+\phi(k-1), \forall k \geq 1$.

We define $f\left(x^{k}, x^{l}\right)=a^{S_{k+l}-S_{k}-S_{l}}$ for $k, l \geq 1$ (observe that if $n \neq \infty$ then $a^{t}$ is well defined for $t \in \mathbb{Z}_{n}$ since $a$ has order $n$ ). 
It is easy to verify that $f\left(x^{k+s m}, x^{l}\right)=f\left(x^{k}, x^{l+t m}\right)=f\left(x^{k}, x^{l}\right),(\forall) s, t \geq 0,(\forall) l, k \geq 1$. Observe also that $f\left(x, x^{k}\right)=a^{\phi(k)}$. This will be useful for the converse.

$f$ is obviously symmetric.

We need to prove that $f$ is a 2-cocycle, that is:

$$
\begin{aligned}
f\left(x^{k}, x^{l}\right) f\left(x^{k+l}, x^{p}\right) & =f\left(x^{l}, x^{p}\right) f\left(x^{k}, x^{l+p}\right) \\
a^{S_{k+l}-S_{k}-S_{l}+S_{k+l+p}-S_{p}-S_{k+l}} & =a^{S_{p+l}-S_{p}-S_{l}+S_{k+l+p}-S_{k}-S_{l+p}} \\
a^{S_{k+l+p}-S_{k}-S_{l}-S_{p}} & =a^{S_{k+l+p}-S_{k}-S_{l}-S_{p}},(\forall) k, l, p \geq 1
\end{aligned}
$$

and the later is clearly true.

So to each $\phi \in \Sigma_{m, n}$ we have associated a symmetric 2-cocycle.

Now suppose $f$ is a symmetric 2-cocycle. Define $\phi_{f}=\phi$ such that $a^{\phi(k)}=f\left(x, x^{k}\right), k \in \mathbb{Z}$. It is obvious that $\phi \in \Sigma_{m, n}$ because $x$ has order $m$ and $f$ is normalized.

Define $S_{k}^{\phi}=S_{k}=\phi_{f}(0)+\ldots+\phi_{f}(k-1)$.

Using the cocycle condition on $f$ and straightforward computation it follows that $f\left(x^{l}, x^{k}\right)=$ $a^{S_{k+l}-S_{k}-S_{l}},(\forall) k, l \geq 1$.

Hence, the map that associates to each cocycle the function $\phi_{f}$ is a bijective map between the cocycles and $\Sigma_{m, n}$.

Proposition 3.5. A crossed product $C_{n} \#^{f} C_{m}, m, n \geq 2$ is a cyclic group if and only if $\left(S_{m}, m, n\right)=1$, where $S_{k}=\phi(0)+\ldots+\phi(k-1), \phi: \mathbb{Z} \rightarrow \mathbb{Z}_{n}, a^{\phi(k)}=f\left(x, x^{k}\right)$ and $x$ is a generator for $C_{m}$.

Remark 3.6. Observe that $S_{m}$ is not a number, but a class (modulo $n$ ); however $\left(S_{m}, m, n\right)$ does not depend on the choice of a representant for $S_{m}$.

Proof. We will prove that $C_{n} \#^{f} C_{m}$ is isomorphic to $C_{n} \#_{i}^{1} C_{m}$ where $i \in\{0,1, \ldots, n-1\}$ such that $S_{m}=i(\bmod n)$. The conclusion will follow from Proposition 3.2.

Let $i$ be the unique representant of $S_{m}$ from $\{0,1, \ldots, n-1\}$.

Denote by $E=<a, b \mid a^{n}=1, b^{m}=a^{i}, a b=b a>$ and by $F=C_{n} \#{ }^{f} C_{m}$ the twisted product associated to the 2-cocycle $f$ (see Example 1.2.3)

Define $\theta: E \rightarrow F$ by $\theta(a)=(a, 1)$ and $\theta(b)=(1, x)$.

It is straightforward to see that $(1, x)^{k}=\left(a^{S_{k}}, x^{k}\right), \forall k \geq 1$ hence $(1, x)^{m}=\left(a^{S_{m}}, x^{m}\right)=$ $\left(a^{i}, 1\right)=(a, 1)^{i}$ and $(1, x)^{k} \notin<a>, \forall k \in\{1, \ldots, m-1\}$. Also $(a, 1)^{n}=1$ and $(a, 1)(1, x)=(a, x)=(1, x)(a, 1)$. That is $(a, 1)$ and $(1, x)$ verify the same relations in $F$ as $a$ and $b$ do in $E$. Hence $\theta$ is a morphism of groups.

Let's observe that $(a, 1)$ and $(1, x)$ generate the group $F$. Indeed consider $\left(a^{u}, x^{k}\right) \in F$. Then $\left(a^{u}, x^{k}\right)=\left(a^{u}, 1\right)\left(1, x^{k}\right)=\left(a^{u-S_{k}}, 1\right)\left(a^{S_{k}}, x^{k}\right)=(a, 1)^{u-S_{k}}(1, x)^{k}$. Therefore the morphism $\theta$ is also surjective and since the groups are finite it is an isomorphism.

Proposition 3.7. A crossed product $C_{g} \#^{f} C_{m}, m \geq 2$, is cyclic iff $\left(S_{m}, m\right)=1$, where $S_{m}=\phi(0)+\ldots+\phi(m-1), \phi: \mathbb{Z} \rightarrow \mathbb{Z}, g^{\phi(k)}=f\left(x, x^{k}\right),<x>=\mathbb{Z}_{m}$. 
Proof. We will prove that $C_{g} \#^{f} C_{m}$ is isomorphic to $E=<g, h \mid h^{m}=g^{S_{m}}, g h=h g>$ hence the conclusion follows from Proposition 3.3 .

Denote by $F=C_{g} \#{ }^{f} C_{m}$ the twisted product associated to the 2-cocycle $f$ (see Example 1.2.3).

Define $\theta: E \rightarrow F$ by $\theta(g)=(g, 1)$ and $\theta(h)=(1, x)$. It is easy to see that $(g, 1)(1, x)=$ $(g, x)=(1, x)(g, 1)$ and $(1, x)^{k}=\left(g^{S_{k}}, x^{k}\right)$. Hence $(1, x)^{m}=\left(g^{S_{m}}, 1\right)=(g, 1)^{S_{m}}$. Therefore $\theta$ is a morphism of groups. Moreover, since $\left(g^{k}, x^{l}\right)=(g, 1)^{k-S_{l}}(1, x)^{l}$ we obtain that $\theta$ is a surjection. Furthermore :

$$
\begin{aligned}
\operatorname{ker}(\theta) & =\left\{g^{k} h^{l}: k, l \in \mathbb{Z}, \theta\left(g^{k} h^{l}\right)=(1,1)\right\} \\
& =\left\{g^{k} h^{l}: k, l \in \mathbb{Z},(g, 1)^{k}(1, x)^{l}=(1,1)\right\} \\
& =\left\{g^{k} h^{l}: k, l \in \mathbb{Z},\left(g^{k+S_{l}}, x^{l}\right)=(1,1)\right\} \\
& =\left\{g^{k} h^{l}: k, l \in \mathbb{Z}, m \mid l, k=-S_{l}\right\} \\
& =\left\{g^{k} h^{l}: k, l \in \mathbb{Z}, l=s m, k=-s S_{m}\right\} \\
& =\left\{\left(g^{-S_{m}} h^{m}\right)^{s}: s \in \mathbb{Z}\right\} \\
& =\{1\}
\end{aligned}
$$

Hence $\theta$ is a bijection.

In conclusion, with the above notations, we proved the following theorem:

Theorem 3.8. A normalized crossed product $E=H \#_{\alpha}^{f} G$ is a cyclic group if and only if one of the following are true:

(1) $H \simeq C_{n}, G \simeq C_{m}$, for some $m, n \geq 2, \alpha$ is trivial and $\left(S_{m}, m, n\right)=1$

(2) $H \simeq C_{g}, G \simeq C_{m}$, for some $m \geq 2, \alpha$ is trivial and $\left(S_{m}, m\right)=1$.

Let us consider a numerical example. Define $\phi \in \Sigma_{3, \infty}$ by $\phi(0)=0, \phi(1)=1, \phi(2)=1$ and consider the corresponding symmetric 2-cocyle $f: \mathbb{Z}_{3} \times \mathbb{Z}_{3} \rightarrow \mathbb{Z}$ (cf. Proposition 3.4).

An easy computation leads us to

$f(\hat{0}, \hat{u})=f(\hat{u}, \hat{0})=f(\hat{2}, \hat{2})=0, \forall \hat{u} \in \mathbb{Z}_{3}$

$f(\hat{1}, \hat{1})=f(\hat{1}, \hat{2})=f(\hat{2}, \hat{1})=1$

Since $S_{3}=2$ it follows from Theorem 3.8 that $\mathbb{Z} \times{ }^{f} \mathbb{Z}_{3} \simeq \mathbb{Z}$.

We can also find the generator of $\mathbb{Z} \times^{f} \mathbb{Z}_{3}$, namely $(0, \hat{2})$. Indeed:

$(0, \hat{2})^{2}=(0+0+f(\hat{2}, \hat{2}), \hat{2}+\hat{2})=(0, \hat{1})$ and

$(0, \hat{2})^{3}=(0+0+f(\hat{1}, \hat{2}), \hat{2}+\hat{1})=(1, \hat{0})$.

\section{ACKNOWLEDGEMENT}

We wish to thank Professor Gigel Militaru, who suggested the problem studied here, for his great support and for the useful comments from which this manuscript has benefitted. 


\section{REFERENCES}

[1] A. Adem, R. J. Milgram, Cohomology of finite groups, Springer, 2nd Edition, 2004. Zbl 1061.20044

[2] A.L. Agore, Constructions in group theory, dizertation, 2008, Univ. of Bucharest (in romanian).

[3] A.L. Agore, G. Militaru, Crossed Product of Groups. Applications, Arabian J. Sci. and Engineering 33(2008), 1-17.

[4] H. Bechtell, The Theory of groups, Addison-Wesley Publishing Company, 1971. Zbl. 0229.20001

[5] P. A. Grillet, Abstract Algebra, Graduate Texts in Mathematics 242, Springer, 2007. Zbl. 1122.00001

[6] O. Hölder, Bildung zusammengesetzter Gruppen, Math. Ann. 46(1895), 321-422.

[7] J. Rotman, An introduction to the theory of groups. Fourth edition. Graduate Texts in Mathematics 148, Springer-Verlag, New York, 1995. Zbl. 0810.20001

[8] C. Weibel, An introduction to homological algebra, Cambridge Univ. Press, 1994. Zbl. 0797.18001

Faculty of Mathematics and Computer Science, University of Bucharest, Str. Academiei 14, RO-010014 Bucharest 1, Romania

E-mail address: ana.agore@fmi.unibuc.ro

E-mail address: dragos.fratila@fmi.unibuc.ro 